\title{
Estresse térmico durante o pré-abate em frangos de corte
}

\author{
Heat stress during the pre-slaughter on broiler chicken
}

\author{
Camila Brossi ${ }^{I}$ Carmen Josefina Contreras-Castillo ${ }^{\text {II }}$ Erik de Almeida Amazonas ${ }^{\text {III }}$ \\ José Fernando Machado Menten ${ }^{\mathrm{IV}}$
}

\section{- REVISÃO BIBLIOGRÁFICA -}

\section{RESUMO}

A cadeia da carne de frango no Brasil é um importante setor do agronegócio, ocupando a terceira maior produção mundial e o primeiro lugar nas exportações. Para atender a demanda, é fundamental minimizar os problemas de manejo, que causam o aparecimento de problemas fisiológicos. A seleção genética intensiva, que é usada na obtenção de taxas de crescimento mais rápidas para frangos de corte, contribuiu para que as linhagens melhoradas atuais sejam muito susceptíveis ao estresse térmico. Quando a temperatura ambiente se eleva acima da zona de termoneutralidade, a ave é submetida a uma condição de estresse, podendo gerar hipertermia aguda, alcalose respiratória, desequilíbrio eletrolítico, redução no consumo de alimento, menor taxa de crescimento, aumento na mortalidade elou até mesmo efeitos negativos na qualidade da carne, pela incidência de carnes pálidas e, em outros casos, escuras, interferindo nas propriedades como rendimento industrial, capacidade de retenção de água, cor e maciez. Os resultados de avaliação dos parâmentros de qualidade observados na literatura evidenciam que o estresse térmico, crônico ou agudo sofrido por frangos de corte geram consequências negativas sobre as propriedades funcionais da carne. São necessários mais estudos sobre essa questão, principalmente em relação ao Brasil, por ser um país tropical, com elevadas temperaturas, especialmente, no verão, para compreender, prevenir e contornar a extensão ou intensidade do estresse, evitando problemas e prejuízos para a cadeia da carne.

Palavras-chave: aves, alterações fisiológicas, músculo, carne.

\begin{abstract}
The chicken meat chain in Brazil is an important sector of agribusiness, accounting for the third largest world's production and first place in exports. To meet this demand, it is essential to minimize management problems, which cause the appearance of physiological problems. The intensive genetic selection used to obtain faster growth rates for broilers contributed for the current improved strains to become very susceptible to heat stress. When the environment temperature rises above the thermoneutrality zone, the bird is submitted to a stress condition, which can generate acute hyperthermia, respiratory alkalosis, electrolyte imbalance, reduction in food consumption, lower growth rate, increased mortality rate and even negative effects on meat quality due to the incidence of pale meat and dark in other cases, interfering in properties such as industrial yield, water holding capacity, color and tenderness. The results of the evaluation of quality parameters observed in literature showed that the heat stress, chronic or acute, suffered by broiler chickens, generates negative consequences on the functional properties of meat. Further studies with this research line should be conducted, mainly in Brazil, for being a tropical country with high temperatures in the summer in order to understand, prevent and overcome the extent or intensity of this stress, avoiding problems and damages to the meat chain.
\end{abstract}

Key words: poultry, physiological alterations, muscle, meat.

'Programa de Pós-graduação em Ciência e Tecnologia de Alimentos, Escola Superior de Agricultura Luiz de Queiroz (ESALQ), Universidade de São Paulo (USP), 13418-900, Piracicaba, SP, Brasil. E-mail: camibro@yahoo.com.br. Autor para correspondência. "Departamento de Agroindústria, Alimentos e Nutrição, ESALQ, USP, Piracicaba, SP, Brasil.

IIIPrograma de Pós-graduação em Ciência Animal e Pastagens, ESALQ, USP, Piracicaba, SP, Brasil.

${ }^{\mathrm{IV}}$ Departamento de Zootecnia, ESALQ, USP, Piracicaba, SP, Brasil. 


\section{INTRODUÇÃO}

O Brasil atingiu, em 2004, a condição de terceiro maior produtor mundial de carne de frango e é o maior exportador desse produto (ABEF, 2008). Essa condição tem sido mantida desde então, com aumento da participação brasileira na liderança do mercado mundial (ABEF, 2008), refletindo a importância do agronegócio do frango para a economia do país.

O frango de corte é um animal doméstico geneticamente aprimorado para rápido crescimento e deposição de tecido muscular. O desempenho produtivo dessas aves atingiu índices impressionantes com os avanços genéticos e nutricionais, resultando em animais com metabolismo mais acelerado (LAGANA, 2005). No entanto, sua capacidade termorreguladora parece ser deficiente para enfrentar condições de altas temperatura e umidade (LAGANA, 2005), o que mostra que os animais selecionados para alto desempenho não são tão adaptados ao ambiente em que vivem como eram seus antepassados (CLASSEN, 2000).

As condições climáticas são as que mais diretamente afetam as aves, por comprometer a manutenção da homeotermia, que é uma função vital (OLIVEIRA et al., 2006). O desequilíbrio fisiológico causado por altas temperaturas e umidade relativa do ar, no momento do abate, tem efeito direto sobre as reservas de glicogênio muscular, responsáveis pelo desenvolvimento das reações bioquímicas post mortem (PETRACCI et al., 2001), que determinarão a qualidade da carne e suas propriedades funcionais (CHEFTEL et al., 1989), ou seja, suas características que possuem implicações tecnológicas diretas e que influenciam decisivamente os aspectos econômicos dos produtos (BIANCHI et al., 2005).

O objetivo desta revisão bibliográfica é esclarecer os efeitos que o estresse térmico pré-abate causa no metabolismo de frangos de corte, com conseqüentes transtornos à qualidade da carne oriunda dessas aves, a fim de demonstrar a importância da condição ambiental sobre a produção eficiente de carne de frango.

\section{Estresse}

O conceito de estresse foi introduzido pelo fisiologista alemão Hans Seyle em 1936, mas ainda hoje é um tema controverso entre as pessoas envolvidas com animais de produção, desde trabalhadores até a comunidade científica, talvez pela relação normalmente estabelecida entre estresse e bem-estar animal.

O termo estresse é uma expressão genérica referente a ajustes fisiológicos, tais como ritmo cardíaco e respiratório, temperatura corporal e pressão sanguínea, os quais ocorrem durante a exposição do animal a condições adversas (HEDRICK et al., 1994). CARRASCO \& Van de KAR (2003) definiram estresse como "respostas organizadas na tentativa de aumentar a sobrevivência do indivíduo e são representadas por alterações das funções autônomas, secreção de múltiplos hormônios e mudanças de comportamento”.

Os principais fatores, no período pré-abate, responsáveis por desencadear alterações fisiológicas características do estresse são: intervalo de jejum e dieta hídrica (SAMS \& MILLS, 1993), transporte (MITCHELL \& KETTLEWELL, 1998) e temperaturas ambientais (SANDERCOCK et al., 2001; DEBUT et al., 2003).

\section{Estresse térmico}

As aves, por serem homeotermas, dispõem de um centro termorregulador localizado no hipotálamo. Esse centro é capaz de controlar a temperatura corporal por meio de mecanismos fisiológicos e de respostas comportamentais, mediante a produção e liberação de calor, possibilitando a manutenção da temperatura corporal normal (MACARI et al., 1994).

Os principais mecanismos ativados para manter a homeostase térmica em frangos são a radiação, a convecção e a evaporação (quase que exclusivamente pela respiração, visto que esses animais não possuem glândulas sudoríparas). Em temperaturas ambientais de até $21^{\circ} \mathrm{C}$, imperam as perdas sensíveis de calor por meio dos processos de radiação, condução e convecção; já em altas temperaturas, a principal rota de dissipação do calor é a evaporação respiratória (HILLMAN et al., 1985).

Normalmente as asas são afastadas do corpo (aumentando a área de superfície), e as penas são eriçadas para permitir o resfriamento corporal. Internamente, a corrente sanguínea é desviada de órgãos como fígado, rins e intestinos para a circulação periférica, permitindo melhor troca de calor. A perda de calor não-evaporativo pode também ocorrer com o aumento da produção de urina, se essa perda de água for compensada pelo maior consumo de água fria (BORGES et al., 2003).

LIN et al. (2005) observaram que há um aumento significativo da temperatura retal, da temperatura da plumagem nas costas e da pele do peito em frangos com quatro semanas de idade expostos a temperaturas acima de $35^{\circ} \mathrm{C}$, corroborando o trabalho anterior de YAHAV et al. (1997), que indicou aumento de carga de calor e distúrbio no balanço térmico das aves. A elevação da temperatura da superfície corporal sugere redução da capacidade em perder calor de forma não-evaporativa (BELAY \& TEETER, 1993). 
A melhor ferramenta de dissipação de calor utilizada pela ave é a hiperventilação (aumento da taxa respiratória). Esse processo remove aproximadamente 540 calorias por grama de água perdida pelos pulmões. No entanto, o processo necessita de grande esforço da musculatura, resultando em um maior aporte de energia e geração de mais calor (BUTCHER \& MILES, 1996). A morte ocorre rapidamente por exaustão, principalmente em aves mais pesadas.

O objetivo da hiperventilação é aumentar a perda de calor pelos mecanismos evaporativos nas vias aéreas superiores. Ocorre simultaneamente vasodilatação periférica, com maior fluxo de sangue nas extremidades e conseqüente perda de calor evaporativa cutânea. Essa perda de calor é fisicamente dependente do gradiente de pressão de vapor do ambiente, ou seja, a gotícula de água somente será evaporada caso a pressão de vapor do meio seja menor do que aquela da via aérea superior ou superfície da pele (MACARI et al, 1994).

De acordo com BELAY \& TEETER (1993), a temperatura ambiental e a umidade relativa (UR) influenciam diretamente a perda de calor sensível e latente do corpo. A capacidade das aves em suportar o calor é inversamente proporcional ao teor de UR do ar, ou seja, quanto maior a UR, maior a dificuldade da ave em remover calor interno pelas vias aéreas, elevando a freqüência respiratória (OLIVEIRA et al., 2006).

A seleção genética intensiva usada para serem obtidas taxas de crescimento mais rápidas para frangos de corte contribuiu para que as linhagens melhoradas atuais sejam muito susceptíveis ao estresse térmico (DEEB \& CAHANER, 2001). A reduzida resistência térmica de frangos de corte pode ser atribuída à baixa capacidade das modernas linhagens melhoradas em perder calor (MacLEOD \& HOCKING, 1993) ou ao aumento impróprio de produção do calor, durante exposição a altas cargas de temperatura (SANDERCOCK et al., 1995).

Um ambiente é considerado confortável para aves adultas quando apresenta temperaturas de 16 a $23^{\circ} \mathrm{C}$ e UR do ar de 50 a $70 \%$; porém, quando a temperatura ambiente se eleva acima da zona de termoneutralidade, a ave é submetida ao estresse térmico (TINÔCO, 1998). A temperatura corporal e taxa de ventilação aumentam, provocando uma redução na pressão parcial de gás carbônico no sangue $\left(\mathrm{PCO}_{2}\right.$, $\mathrm{mmHg}=49,34$ em condições termoneutras e 46,03 sob estresse térmico, segundo BORGES et al., 2003), levando a uma queda na concentração de íons bicarbonato $\left(\mathrm{HCO}_{3}^{-}\right)$e hidrogênio $\left(\mathrm{H}^{+}\right)$, o que caracteriza um desequilíbrio denominado alcalose respiratória. Nessas condições, ocorre uma compensação renal com aumento da excreção de $\mathrm{HCO}_{3}^{-}$e redução de excreção de $\mathrm{H}^{+}$pelos rins, procurando ajustar o balanço ácidobase. Em condição crônica de elevada temperatura, esses mecanismos compensatórios não são suficientes, e o desequilíbrio eletrolítico gerado provoca redução no consumo de alimento e, com isso, menor taxa de crescimento, além de aumento na mortalidade das aves (TEETER et al.,1985).

O sódio $\left(\mathrm{Na}^{+}\right)$, o potássio $\left(\mathrm{K}^{+}\right)$e o cloro $\left(\mathrm{Cl}^{-}\right)$ são íons fundamentais na manutenção da pressão osmótica e equilíbrio ácido-base dos líquidos corporais. Porém, durante o estresse térmico, ocorre diminuição dos níveis plasmáticos de $\mathrm{K}^{+}$devido a um aumento na excreção desse íon. A taxa de excreção de $\mathrm{K}^{+}$pela urina é variável, estando ligada à concentração plasmática de $\mathrm{Na}^{+}$e ao estado de hidratação da ave, e as perdas podem ser causadas por um aumento no consumo de água, já que o gradiente osmótico favorece o movimento de água do fluído intracelular para urina, podendo carrear o K ${ }^{+}$(BELAY \& TEETER, 1993). Segundo BORGES et al. (2004), a explicação para esse fenômeno pode ser conhecida como hemodiluição. Além disso, os tecidos passam a liberar $\mathrm{K}^{+}$para a corrente sanguínea, devido à alteração na permeabilidade das membranas, podendo provocar, até mesmo, sinais neurológicos.

Em uma reação de feedback, os fatores ácido-base, especialmente o $\mathrm{pH}$, afetam os processos metabólicos e são importantes, principalmente, para a cadeia de produção/estoque de energia (OLANREWAJU et al., 2006), as taxas de crescimento, a conversão alimentar, o apetite, o desenvolvimento ósseo (PATIENCE, 1990), a imunidade e a sobrevivência dos frangos (MACARI et al., 1994). Dessa forma, supõe-se que o estresse fisiológico associa-se às alterações do perfil ácido-base (SANDERCOCK et al., 2001; BORGES et al., 2003; 2004), no entanto, a interpretação das desordens no balanço ácido-base durante o estresse é complexa, já que muitas variáveis relevantes são alteradas simultaneamente e, em muitas instâncias, em direções opostas, dependendo da espécie e do tipo de agente estressante (OLANREWAJU et al., 2006). Nesse sentido, a hipertermia aguda e a alcalose respiratória em frangos são consideradas causadoras de efeitos deletérios para a produção eficiente de carne de frango e para um rendimento industrial satisfatório (HOWLINDER \& ROSE, 1989).

\section{Estresse térmico crônico}

Em um estresse crônico, quando os limites de conforto são ultrapassados, podem ocorrer síndromes classificadas como: 1) neurogênica (primeiro estágio - reação de alarme); 2) endócrina (estágio de 
resistência à adaptação) e 3) depleção metabólica (estágio de exaustão). Segundo GRANDIN (1998), a presença de corticosterona em aves, liberada em função do estresse de longo prazo, produz muitos sintomas como doenças cardiovasculares (arteriosclerose), ascite e modificações nas funções imunológicas.

O verão, por causa da alta umidade, sempre acompanhada de altas temperaturas, é um desafio para a termorregulação e o bem-estar dos frangos de corte, podendo interferir negativamente na produtividade e qualidade da criação. Isso se deve principalmente ao aumento da mortalidade, à diminuição da ingestão de água e de alimento e, conseqüentemente, à piora na conversão alimentar.

O trabalho de BIANCHI et al. (2007) evidenciou que a incidência de carnes pálidas e com propriedades funcionais prejudicadas é maior no verão, demonstrando a influência do calor para a ocorrência da baixa qualidade em carne de frangos.

Portanto, os produtores devem estar atentos em controlar esses fatores estressantes para que as aves possam manter suas funções fisiológicas normais e consigam produzir carne com qualidade e eficiência.

\section{Estresse térmico agudo}

Frangos expostos a um estresse térmico agudo ou de curto prazo, imediatamente antes do abate, apresentam alterações na qualidade de carne (SANDERCOCK et al., 2001). Episódios de estresse agudo podem ser vivenciados em uma produção comercial ou durante algumas das etapas da cadeia produtiva, como o transporte ou o tempo de espera no frigorífico antes do abate, em que podem ocorrer condições de calor intensas (MITCHELL \& KETTLEWELL, 1998).

A primeira reação de frangos submetidos a um ambiente de temperatura extremamente alta é a elevação de sua temperatura corporal, podendo ser usada como um indicador da extensão do esforço termorregulatório (HOCKING et al., 1994). De acordo com EDENS (1978), frangos expostos a essas condições (por exemplo, a $43^{\circ} \mathrm{C}$ ) mostram logo no início (antes de 90 minutos) aumento na concentração de corticosterona no plasma, depois uma queda significativa, caracterizando a síndrome de insuficiência cortical adrenal aguda (Figura 1). Finalmente, as aves desenvolvem hipertermia severa, caracterizada por aumento do $\mathrm{pH}$ sanguíneo, indicando presença de alcalose respiratória (HOCKING et al., 1994; SANDERCOCK et al., 2001).

É possível que o estresse agudo pré-abate cause, ao mesmo tempo, alterações na permeabilidade das membranas e mudanças no metabolismo do músculo dos frangos, influenciando a qualidade de carne no período post mortem (SANDERCOCK et al., 2001).

Estresse térmico animal versus qualidade de carne

Atualmente, os animais altamente eficientes em termos de produtividade têm demonstrado uma maior susceptibilidade a certas enfermidades e menor resistência aos fatores estressantes (CLASSEN, 2000). Animais com desenvolvimento muscular acelerado podem ter alterações na velocidade da glicólise, o que pode acarretar alterações nas características de qualidade da carne (SANDERCOCK et al., 2001).

Grande parte dessas alterações pode ser relacionada com a aceleração do metabolismo ocorrido em razão do aumento da exigência metabólica dos animais selecionados para a produção de carne. A energia no tecido muscular é armazenada na forma de adenosina trifosfato (ATP), creatina fosfato e glicogênio. Esse último constitui-se na maior reserva energética do músculo (SWATLAND, 1995). Em processos fisiológicos, com disponibilidade de oxigênio, a degradação do glicogênio se dá por meio da glicólise aeróbica, na qual o ácido pirúvico produzido continua com o ciclo dos ácidos tricarboxílicos, formando, em última instância, $\mathrm{CO}_{2}$ e $\mathrm{H}_{2} \mathrm{O}$ e liberando energia na forma de ATP. Já em condiçõ̃es de falta de oxigenação (como o período post mortem), a degradação do glicogênio muscular ocorre via glicólise anaeróbica e leva à formação de ácido láctico a partir do piruvato, reduzindo o pH muscular (Figura 2). Essa redução é necessária para uma correta maturação da carne no processo denominado conversão do músculo em carne.

Essa queda de $\mathrm{pH}$ na carne se dá, geralmente, de valores iniciais de $\mathrm{pH}$ em torno de 7,2 até valores finais ao redor de 5,8. Entretanto, aves estressadas usam rapidamente suas reservas de glicogênio, o que pode resultar em seu esgotamento in vivo, impossibilitando a queda do $\mathrm{pH}$ post mortem, ou ainda, sofrem uma aceleração da glicólise logo após o abate, gerando acúmulo de ácido lático no músculo, com conseqüente diminuição acelerada do pH (MCKEE \& SAMS, 1998).

A depleção das reservas de glicogênio muscular enquanto o animal encontra-se ainda vivo faz com que não haja acúmulo de ácido láctico no músculo post mortem. Assim sendo, o pH final da carne permanecerá elevado, ficando bastante acima do ponto isoelétrico das principais proteínas musculares (actina, pI = 4,7; miosina, pI = 5,4) (BERG, 2001). Nessas condições, a capacidade dessas proteínas em reter água estará bastante alta (DRANSFIELD \& SOSNICKI, 1999), 


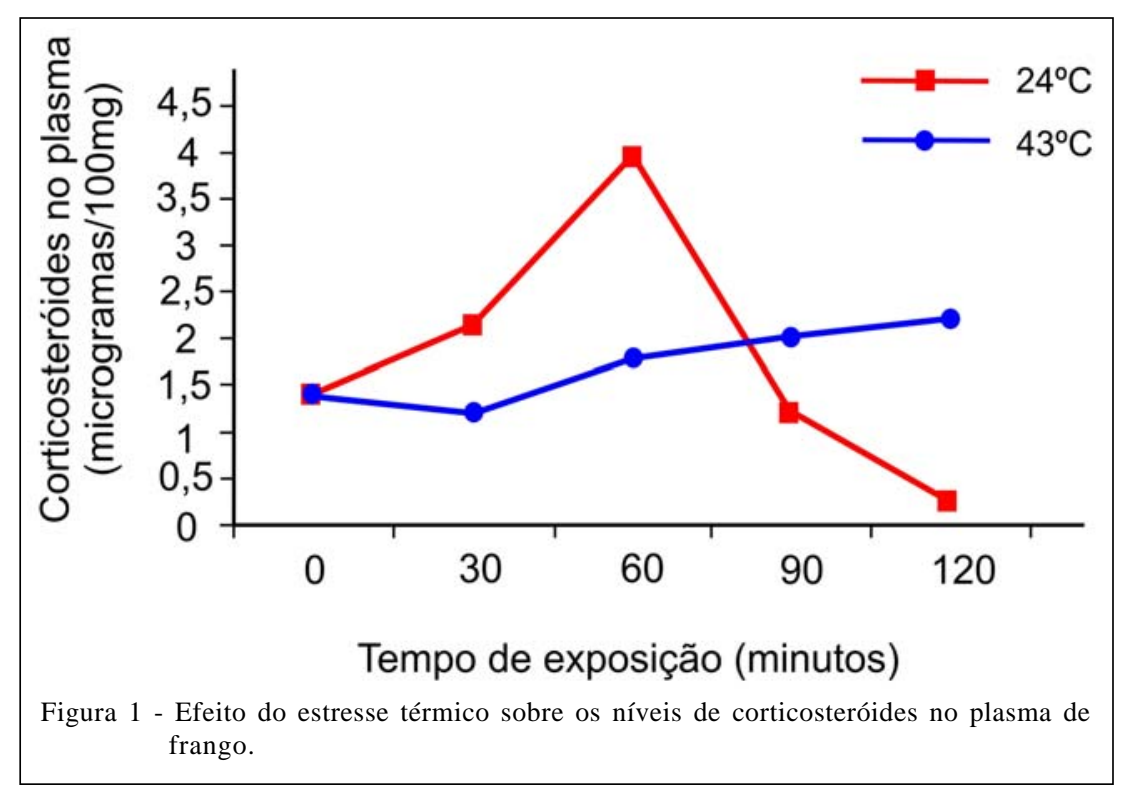

a ponto da água retida no músculo ser praticamente imobilizada, não permitindo seu extravasamento (ANADÓN, 2002). Desse modo, verifica-se uma condição de carne seca, que não é exatamente verdadeira se for levada em consideração a quantidade de água retida pelo músculo. Esse tipo de carne apresenta-se mais rígida que o normal, porque as fibras estão intumescidas pelo preenchimento com fluidos sarcoplasmáticos e também é mais escura, já que devido ao $\mathrm{pH}$ elevado, sua superfície dispersa menos luz do que o normal (LAWRIE, 1998). Geralmente, os consumidores evitam carnes com coloração escura no varejo porque associam sua aparência com a de carnes de animais velhos ou dispostas à venda por um longo tempo (FLETCHER, 2002). Além disso, graças ao $\mathrm{pH}$ próximo do fisiológico, esse tipo de carne constitui-se em um meio propício para o crescimento de microrganismos indesejáveis, reduzindo a vida útil do alimento.

Por outro lado, músculos que apresentam valores de $\mathrm{pH}$ menores que 5,8 em torno de 15 minutos após o abate, quando a carcaça ainda se encontra em temperaturas próximas da fisiológica, geralmente sofrem uma desnaturação parcial das proteínas que o compõem (BARBUT, 1998; TANKSON et al., 2001). A dispersão de luz de um músculo é diretamente proporcional ao grau de desnaturação protéica (ANADÓN, 2002). Quanto maior a desnaturação de suas proteínas, menos luz consegue ser transmitida por entre as fibras musculares, acabando por se dispersar. A luz, quando dispersa, não é capaz de irradiar as mioglobinas contidas dentro do citoplasma das células musculares (o sarcolema), conferindo assim uma tonalidade pálida à carne.
Além disso, o baixo pH ainda influencia a capacidade de retenção de água da carne, uma das principais características de rendimento no processamento do produto e de maciez. OFFER \& KNIGHT (1988) afirmam que a queda de $\mathrm{pH}$ post mortem altera a composição celular e extracelular das miofibrilas, reduzindo a capacidade de reter água nas proteínas musculares. Isso é uma conseqüência da desnaturação protéica, especialmente da miosina. Uma vez desnaturada, a proteína perde suas características físico-químicas e, conseqüentemente, a eficiência de ligação com as moléculas de água torna-se bastante prejudicada.

Esse tipo de carne, por sua cor pálida e, principalmente por sua propriedade de não reter água como um músculo normal, causa transtornos à industrialização. Ela apresenta rendimento deficiente, quando processada, dificuldade em manter sua própria água, baixa absorção de salmoura durante a marinação (WOELFEL et al., 2002), perda de líquido por gotejamento (exsudação) na embalagem, baixa capacidade de emulsificação, pouca coesividade e perda de peso pós-cocção. Com isso, observa-se reduzida suculência (FLETCHER, 2002), além de menor vida útil do produto (BARBUT, 2002).

De acordo com DRANSFIELD \& SOSNICKI (1999), as carnes pálidas possuem também menor potencial proteolítico post mortem, o que contribui para a diminuição da maciez da carne. Segundo esses autores, o rápido declínio de $\mathrm{pH}$ e as altas temperaturas da carcaça inativam o sistema calpaína e reduzem o amaciamento post mortem. 


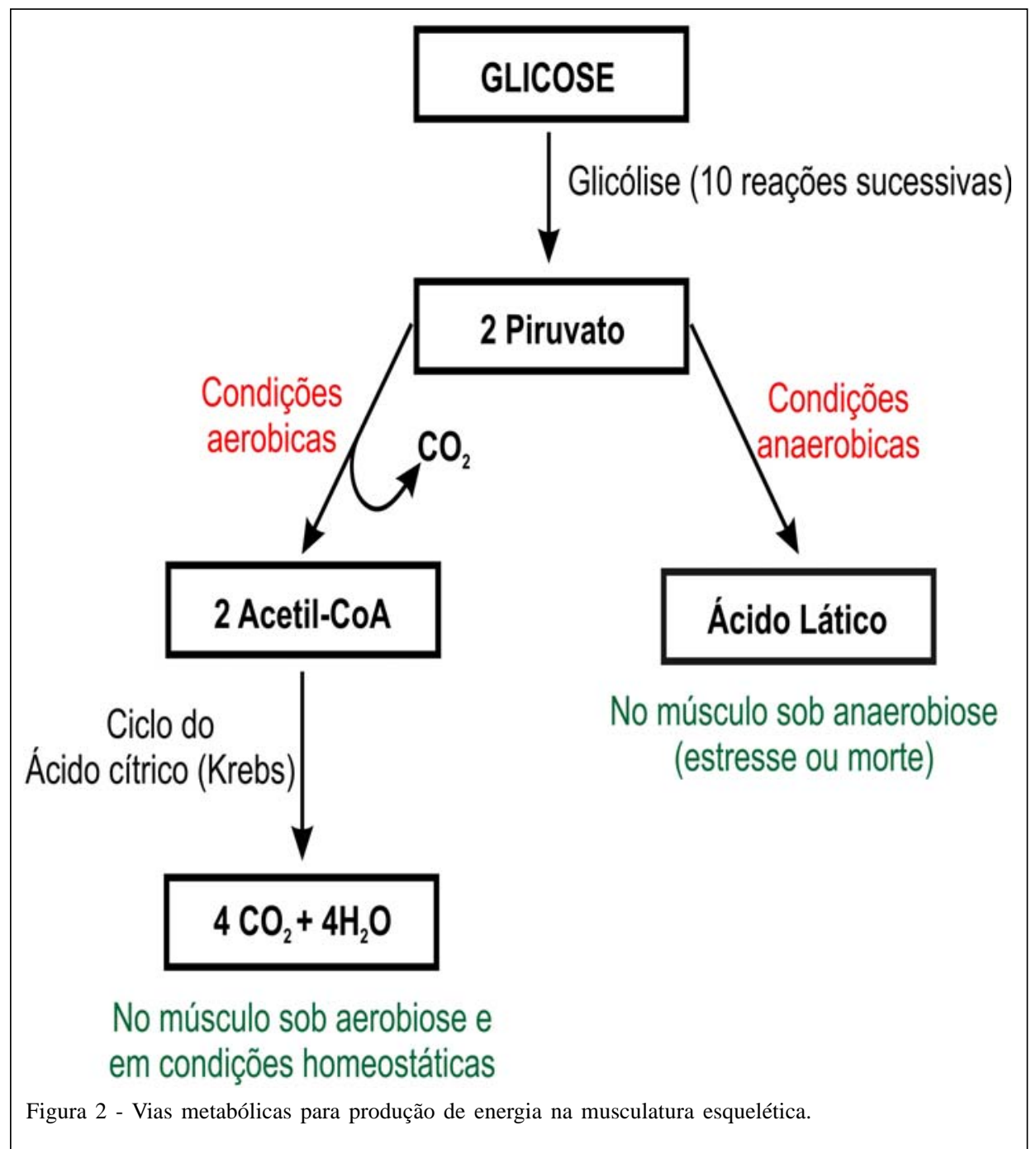

Influência do estresse térmico pré-abate sobre atributos de qualidade de carne

HOLM \& FLETCHER (1997) estudaram a influência do estresse térmico sobre parâmetros de qualidade de carne em frangos e observaram que um estresse de 12 horas, antes do abate, induziu um $\mathrm{pH}$ final abaixo do normal, uma menor de perda de peso por cocção (PPC), assim como um aumento da rigidez da carne de peito de frango (PETRACCI et al., 2001). Com o objetivo de caracterizar respostas fisiológicas e analisar a qualidade de carne de aves expostas a um estresse térmico agudo, SANDERCOCK et al. (2001) realizaram um estudo com dois tratamentos: controle $\left(21^{\circ} \mathrm{C}\right.$ e $50 \%$ UR) e estresse térmico agudo $\left(32^{\circ} \mathrm{C}\right.$ e $75 \%$ UR), ambos em câmara climática por duas horas. Os pesquisadores encontraram maior perda de peso por gotejamento (PPG) e forte efeito do estresse térmico sobre a taxa de queda do $\mathrm{pH}$ post mortem do músculo, sugerindo uma aceleração do metabolismo glicolítico peri mortem em aves estressadas. No entanto, assim como para os estudos citados anteriormente, não foram observados efeitos dos tratamentos, quanto às escalas de cor da carne do peito (Tabela 1). Da mesma forma, BRESSAN \& BERAQUET (2002), a fim de avaliar o efeito da temperatura ambiental pré-abate sobre o desenvolvimento das reações bioquímicas post mortem e a qualidade da carne de peito de frango, realizaram dois ensaios: o primeiro com temperatura de $30^{\circ} \mathrm{C}$ (estresse térmico) e o segundo com temperatura de $17^{\circ} \mathrm{C}$ (conforto térmico). Aves submetidas à temperatura ambiental pré-abate de $30^{\circ} \mathrm{C}$ apresentaram, no músculo Pectoralis major, $\mathrm{pH}$ inicial (15 minutos post mortem) mais baixo que o controle, evidenciando que os frangos submetidos ao estresse tiveram reações de glicólise e hidrólise de ATP mais rápidas do que os frangos mantidos a $17^{\circ} \mathrm{C}$ (Tabela 1$)$.

Os autores DEBUT et al. (2003) avaliaram diferentes condições de estresse pré-abate e reportaram

Ciência Rural, v.39, n.4, jul, 2009. 
Tabela 1 - Diferentes condições de estresse térmico e suas conseqüências sobre os parâmetros de qualidade de carne: pH, cor (L* luminosidade), FC (força de cisalhamento), PPC (perda de peso por cocção) e PPG (perda de peso por gotejamento).

\begin{tabular}{|c|c|c|c|c|c|}
\hline Autores & $\begin{array}{c}\text { HOLM \& } \\
\text { FLETCHER (1997) }\end{array}$ & $\begin{array}{l}\text { PETRACCI et al. } \\
\text { (2001) }\end{array}$ & $\begin{array}{l}\text { SANDERCOCK et al. } \\
\text { (2001) }\end{array}$ & $\begin{array}{c}\text { BRESSAN \& } \\
\text { BERAQUET (2002) }\end{array}$ & DEBUT et al. (2003) \\
\hline Condições & $\begin{array}{c}7^{\circ} \mathrm{C} / 18^{\circ} \mathrm{C} / 29^{\circ} \mathrm{C} \\
\text { por } 12 \text { horas }\end{array}$ & $\begin{array}{c}25^{\circ} \mathrm{C} / 29,5^{\circ} \mathrm{C} / 34^{\circ} \mathrm{C} \\
\text { por } 12 \text { horas }\end{array}$ & $\begin{array}{c}21^{\circ} \mathrm{C} 50 \% \text { UR } \\
32^{\circ} \mathrm{C} 75 \% \text { UR } \\
\text { por } 2 \text { horas }\end{array}$ & $\begin{array}{c}17^{\circ} \mathrm{C} / 30^{\circ} \mathrm{C} \\
\text { da granja ao abate }\end{array}$ & $\begin{array}{c}\text { Controle } / 35^{\circ} \mathrm{C} \\
\text { por } 2 \text { horas }\end{array}$ \\
\hline $\mathrm{pH}$ & $\begin{array}{c}5,84\left(18^{\circ} \mathrm{C}\right) \\
\text { vs. } \\
5,74\left(29^{\circ} \mathrm{C}\right)\end{array}$ & NS & $\begin{array}{c}5,83\left(21^{\circ} \mathrm{C}\right) \\
\text { vs. } \\
5,64\left(32^{\circ} \mathrm{C}\right)\end{array}$ & $\begin{array}{c}6,08\left(17^{\circ} \mathrm{C}\right) \\
\text { vs. } \\
5,98\left(30^{\circ} \mathrm{C}\right)\end{array}$ & $\begin{array}{c}6,13 \text { (controle) } \\
\text { vs. } \\
6,07\left(35^{\circ} \mathrm{C}\right)\end{array}$ \\
\hline Cor $\left(\mathrm{L}^{*}\right)$ & NS & NS & NS & - & $\begin{array}{c}50,31 \text { (controle) } \\
\text { vs. } \\
51,36\left(35^{\circ} \mathrm{C}\right)\end{array}$ \\
\hline $\mathrm{FC}(\mathrm{kg} / \mathrm{g})$ & $\begin{array}{c}3,48\left(18^{\circ} \mathrm{C}\right) \\
\text { vs. } \\
4,18\left(29^{\circ} \mathrm{C}\right)\end{array}$ & $\begin{array}{c}6,05\left(25^{\circ} \mathrm{C}\right) \\
\text { vs. } \\
7,94\left(34^{\circ} \mathrm{C}\right)\end{array}$ & - & NS & - \\
\hline PPC (\%) & $\begin{array}{c}28,27\left(18^{\circ} \mathrm{C}\right) \\
\text { vs. } \\
27,26\left(29^{\circ} \mathrm{C}\right)\end{array}$ & $\begin{array}{c}28,6\left(25^{\circ} \mathrm{C}\right) \\
\text { vs. } \\
27,0\left(34^{\circ} \mathrm{C}\right)\end{array}$ & - & $\begin{array}{c}27,2\left(17^{\circ} \mathrm{C}\right) \\
\text { vs. } \\
28,7\left(30^{\circ} \mathrm{C}\right)\end{array}$ & NS \\
\hline PPG (\%) & - & - & $\begin{array}{c}2,0\left(21^{\circ} \mathrm{C}\right) \\
\text { vs. } \\
3,7\left(32^{\circ} \mathrm{C}\right)\end{array}$ & - & - \\
\hline
\end{tabular}

Todos os resultados expressos na tabela diferem significativamente $(\mathrm{P} \leq 0,05)$ entre os tratamentos mostrados entre parênteses. NS = não apresentou diferença significativa entre os tratamentos avaliados.

- = parâmetro não analisado no experimento.

que o estresse de duas horas a $35^{\circ} \mathrm{C}$ influenciou o $\mathrm{pH}$ e o valor $\mathrm{L}^{*}$ (luminosidade) da carne de frango, mas isso não pôde ser verificado no músculo do peito e, sim, nos músculos da coxa. Segundo esses autores, a carne da coxa mostrou-se mais sensível ao estresse que a do peito. Essa diferença quanto à sensibilidade já foi previamente sugerida pelo estudo genético de LE BIHAN-DUVAL et al. (2003) em qualidade de carne de peru, no qual os efeitos dos fatores ambientais foram mais dominantes para alguns parâmentros avaliados em coxa (pH e cor), pois suas características hereditárias são extremamente mais baixas. $\mathrm{O}$ pH final da carne da coxa foi significativamente mais baixo, e o valor de L*, mais elevado, ou seja, carne mais pálida para o tratamento submetido ao estresse do que para o tratamento controle (Tabela 1).

Os resultados de avaliação dos parâmentros de qualidade observados na literatura, portanto, evidenciam que o estresse térmico sofrido por frangos de corte, no período pré-abate, geram consequências negativas sobre o $\mathrm{pH}$, que tende a ser inferior na carne de animais estressados. Como consequência, os frangos de corte podem sofrer desnaturação protéica e com isso redução da capacidade em reter água nas proteínas musculares. A baixa capacidade de retenção de água pode ser verificada nos valores elevados de perda de peso por cocção (PPC) e perda de peso por gotejamento (PPG), característica indesejável para produtos cárneos, já que esse tipo de produto apresenta rendimento deficiente e pouca suculência. Além disso, as taxas mais elevadas de força de cisalhamento contribuem para a redução da qualidade desse tipo de carne, isso porque apresentam menor potencial proteolítico post mortem, o que leva a uma diminuição da maciez.

ALTAN et al. (2003) testaram a influência do estresse térmico sobre o nível de oxidação das células vermelhas em sangue de frangos de corte submetidos a $38^{\circ} \mathrm{C}$, por três horas. Segundo esses autores, a taxa de peroxidação para os frangos do grupo controle foi $2,01 \mathrm{nmoles} \mathrm{ml}^{-1}$, enquanto que para os frangos submetidos ao estresse foi de 2,59nmoles $\mathrm{ml}^{-1}$, 
ou seja, como conseqüência do estresse térmico, os frangos apresentaram um aumento da geração de radicais livres no sangue. Da mesma forma, LIN et al. (2006) investigaram frangos expostos a um estresse térmico agudo $\left(32^{\circ} \mathrm{C}\right.$ por seis horas). Os resultados desse estudo sugeriram que a elevada temperatura corporal pode induzir alterações metabólicas que envolvem o estresse oxidativo. Os níveis de TBARS (Substâncias Reativas ao Ácido Tiobarbitúrico - teste que quantifica o malonaldeído, um dos principais produtos da decomposição dos hidroperóxidos de ácidos graxos poliinsaturados, formado durante o processo oxidativo) no plasma e no fígado se elevaram em comparação com os frangos controle, assim como nos resultados anteriores de MAHMOUD \& EDENS (2003). A produção de níveis excessivos de espécies reativas ao oxigênio (ROS), enquanto o animal ainda está vivo, pode ser estimulada por condições estressantes ou clínicas (DRÖGE, 2002; MAHMOUD \& EDENS, 2003), resultando em distúrbios no balanço entre a oxidação e os sistemas antioxidantes de defesa, causando peroxidação lipídica e injúrias oxidativas às proteínas e ao DNA(DRÖGE, 2002). Altas temperaturas afetam, de forma adversa, a estrutura e a fisiologia das células, causando prejuízos na transcrição do RNA e, conseqüentemente, das estruturas e funções das membranas e do metabolismo oxidativo (MAGER \& De KRUIJFF, 1995).

A oxidação lipídica é uma das maiores causas de deterioração da qualidade em carnes, contribuindo para degradação do flavor e redução da vida útil dos produtos devido à iniciação da peroxidação (VERCELLOTTI et al., 1992). Porém, segundo BROSSI et al. (2007), o estresse térmico induzido de frangos de corte, em câmara climática por duas horas a aproximadamente $35^{\circ} \mathrm{C}$ e $75 \%$ de umidade relativa, não provocou alteração química detectada pela análise de TBARS em músculo da sobrecoxa, refrigerada durante um, sete ou 14 dias de armazenamento. Esses resultados sugerem que a análise de TBARS realizada na carne in natura pode não ter a mesma sensibilidade que quando realizada no sangue ou fígado, ou ainda, que os efeitos desse perfil de estresse aplicado não apresentaram variação quanto à oxidação lipídica na carne.

\section{CONSIDERAÇÕES FINAIS}

Um dos maiores desafios para a indústria de carnes é oferecer produtos padronizados, com aparência, textura e sabor agradáveis e estáveis durante toda a vida útil desses produtos, com inocuidade e o menor custo possível. Dentro desse aspecto, o estudo e o conhecimento das propriedades funcionais das matérias-primas e dos fatores que as influenciam, com especial atenção ao estresse pré-abate, são imprescindíveis para garantir resultados positivos para toda a cadeia produtiva de carne de frango. A pesquisa sobre o estresse animal relacionando-o com a qualidade de carne favorece a indústria avícola que passa a ter um direcionamento para se adequar às exigências da indústria processadora, a qual passa a obter melhores resultados, maior rendimento, propriedades funcionais asseguradas e melhor aceitação dos produtos pelo mercado consumidor.

Já se sabe que o esforço da ave sob condição de estresse térmico causa transtornos quanto à interferência na qualidade dos atributos da carne; porém, são necessários mais estudos sobre essa questão, já que os resultados obtidos até o momento ainda são pouco esclarecedores. É preciso compreender como a extensão ou a intensidade do estresse pode originar respostas tão variáveis, efeitos e conseqüências tão distintos e, com isso, prevenir ou contornar os possíveis problemas e prejuízos na qualidade de carne.

\section{AGRADECIMENTO}

Agradecimentos à Fundação de Amparo à Pesquisa do Estado de São Paulo (FAPESP), pelo financiamento do projeto de pesquisa "Qualidade da carne de frangos de corte: efeitos de genética, estresse pré-abate e nutrição”.

\section{REFERÊNCIAS}

ABEF - ASSOCIAÇÃO BRASILEIRA DOS PRODUTORES E EXPORTADORES DE CARNE DE FRANGO. Estatísticas da produção. Capturado em 20 jul. 2008. On-line. Disponível na Internet: http://www.abef.com.br

ALTAN, O. et al. Effect of heat stress on oxidative stress, lipid peroxidation and some stress parameters in broilers. British Poultry Science, London, v.44, n.4, p.545-550, 2003. Disponível em: http://www.informaworld.com/smpp/ content $\sim \mathrm{db}=$ all ? content $=10.1080 / 00071660310001618334$. Doi: 10.1080/00071660310001618334.

ANADÓN, H.L.S. Biological, nutritional and processing factors affecting breast meat quality of broilers. 2002. 171f. Thesis (Doctor of Philosophy in Animal and Poultry Sciences) - Faculty of Virginia Polythecnic Institute and State University.

BARBUT, S. Estimating the magnitude of the PSE problem in poultry. Journal of Muscle Food, Malden, v.9, p.35-49, 1998. Disponível em: http://www3.interscience.wiley.com/ journal/119943956/abstract?CRETRY=1\&SRETRY=0. Doi: 10.1111/j.1745-4573.1998.tb00642.x.

BARBUT, S. Meat color and flavor. In: BARBUT, S. Poultry products processing: an industry guide. New York: CRC, 2002. Chap.13, p.429-463. 
BELAY, T.; TEETER R.G. Broiler water balance and thermobalance during thermoneutral and high ambient temperature exposure. Poultry Science, Ithaca, v.72, p.116124, 1993.

BERG, E.P. Influence of stress on composition and quality of meat poultry, and meat products. 2001. Capturado em 12 nov. 2006. On line. Disponível na Internet: http:// www.fass.org/fass01/pdfs/Berg.pdf

BIANCHI, M. et al. Physical and functional properties of whole and ground pale broiler breast meat. Poultry Science, Ithaca, v.84, p.803-808, 2005.

BIANCHI, M. et al. The influence of the season and market class of broiler chickens. Poultry Science, Ithaca, v.86, p.959963, 2007.

BORGES, S.A. et al. Physiological responses of broiler chickens to heat stress and dietary electrolyte balance (sodium plus potassium minus chloride, milliequivalents per kilogram). Poultry Science, Ithaca, v.83, p.1551-1558, 2004.

BORGES, S.A. et al. Dietary electrolyte balance for broiler chickens exposed to thermoneutral or heat-stress environments. Poultry Science, Ithaca, v.82, p.428-435, 2003.

BRESSAN, M.C.; BERAQUET, N.J. Efeito de fatores préabate sobre a qualidade da carne de peito de frango. Ciência e Agrotecnologia, Lavras. v.26, n.5 p.1049-1059, 2002.

BROSSI, C. et al. Oxidação lipídica em carne de frango exposto a estresse térmico severo pré-abate. In: CONGRESSO BRASILEIRO DE CIÊNCIA E TECNOLOGIA DE CARNES, 4., 2007, Campinas, SP. Anais... Campinas: Centro de Pesquisa e Desenvolvimento de Carnes - Instituto de Tecnologia de Alimentos, 2007. V.1. p.231-233.

BUTCHER, G.D.; MILES, R. Heat stress management in broilers. Institute of Food and Agricultural Sciences, VM 65, University of Florida, 1996. Capturado em: 20 jul. 2007. Online. Disponível na Internet: http://www.edis.ifas.ufl.edu

CARRASCO, G.A.; van de KAR, L.D. Neuroendocrine pharmacology of stress. European Journal of Pharmacology, Amsterdam, v.463, p.235-272, 2003.

CHEFTEL, J.C. et al. Proteinas alimentarias: bioquímica propriedades funcionales - valor nutritivo - modificaciones químicas. (Tradução de Francisco López Capont). Zaragoza: Acribia, 1989. 346p.

CLASSEN, H.L. Managing metabolic disease in rapidly growing strains of poultry. In: HILL, W.G. et al. The challenge of genetic change in animal production. Edinburgh: Journal of the British Society of Animal Science, 2000. p.63-64. (Occasional publication, 27).

DEBUT, M. et al. Variation of chicken technological meat quality in relation to genotype and preslaughter stress conditions. Poultry Science, Ithaca, v.82, p.1829-1838, 2003.

DEEB, N.; CAHANER, A. Genotype-by-environment interaction with broiler genotypes differing in growth rate: 2 . The effects of high ambient temperature on dwarf versus normal broilers. Poultry Science, Ithaca, v.80, p.541-548, 2001.
DRANSFIELD, E.; SOSNICKI, A.A. Relationship between muscle growth and poultry meat quality. Poultry Science, Ithaca, v.78, p.743-746, 1999.

DRÖGE, W. Free radicals in the physiological control of cell function. Physiological Reviews, Baltimore, v.82, n.1, p.4795, 2002.

EDENS, F.W. Adrenal cortical insufficiency in young chickens exposed to a high ambient temperature. Poutry Science, Ithaca, v.57, p.1746-1750, 1978.

FLetCher, D.L. Poultry meat quality. World's Poultry Science Journal, Ithaca, v.58, n.2, p.131-145, 2002.

GRANDIN, T. Objective scoring on animal handling and stunning practices in slaughter plants. Journal of The American Veterinary Medical Association, Chicago, v.212, p.36-39, 1998.

HEDRICK, H.B. et al. Principles of meat science. 3.ed. Dubuque: Kendall/Hunt Publishing, 1994. 354p.

HILLMAN, P.E. et al. Physiological responses and adaptations to hot and cold environments. In: YOUSEF, M.K. Stress physiology in livestock. Boca Raton: CRC, 1985. 3v. p.171.

HOCKING, P.M. et al. Haematology and blood composition at two ambient temperatures in genetically fat and lean adult broiler breederfemales fed ad libitum or restricted throughout life. British Poultry Science, London, v.35, p.799-807, 1994.

HOLM, C.G.P.; FLETCHER, D.L. Antemortem holding temperatures and broiler breast meat quality. Journal of Applied Poultry Research, Athens, v.6, p.180-184,1997.

HOWLINDER, M.A.R.; ROSE, S.P. Rearing temperature and meat yield of broilers. British Poultry Science, London, v.34, p.925-938, 1989.

LAGANA, C. Otimização da produção de frangos de corte em estresse por calor. 2005. 180f. Tese (Doutorado em Zootecnia) - Universidade Federal do Rio Grande do Sul, Porto Alegre.

LAWRIE, R.A. The conversion of muscle to meat. In: LAWRIE, R.A. Lawrie's meat science. 6.ed. Cambridge: Woodhead, 1998. p.96-118.

LE BIHAN-DUVAL, E. et al. Genetic parameters of meat technological quality traits in a grand-parental commercial line of turkey. Genetics, Selection, Evolution, Paris, v.35, p.623635, 2003. Disponível em: http://www.gse-journal.org/ index.php?option=article \&access $=$ doi\&doi $=10.1051 /$ gse:2003043. Doi: 10.1051/gse:2003043.

LIN, H. et al. Acute heat stress induces oxidative stress in broiler chickens. Comparative Biochemistry and Physiology. Part A, Oxford, v.144, p.11-17, 2006. Disponível em: http:// www.sciencedirect.com/science?_ob=ArticleURL\&_udi=B6VNH4JDMVDY-2\&_user $=687358$ \&_rdoc $=1 \&$ \&_mt $=$ \&_orig $=$ search $\&_{\text {_ s ort }}=\mathrm{d} \&$ vi e w $=$ c $\&_{\text {_ }}$ a c c t $=$ C $000037899 \&$ _ v ersion $=1 \&$ _url Version $=0 \&$ _us e ri d $=68735$ 8\&md5=0593b3ecd832f4e4e9ccbc43711032b7. Doi: 10.1016/ j.cbpa.2006.01.032.

Ciência Rural, v.39, n.4, jul, 2009. 
LIN, H. et al. Thermoregulation responses of broiler chickens to humidity at different ambient temperatures ii: four weeks of age. Poultry Science, Ithaca, v.84, p.1173-1178, 2005.

MACARI, M. et al. Fisiologia aviária aplicada a frangos de corte. Jaboticabal: Fundação de Estudos e Pesquisas em Agronomia Medicina Veterinária e Zootecnia - FUNEP, 1994. $296 p$.

MacLEOD, M.G.; HOCKING, P.M. Thermoregulation at high ambient-temperature in genetically fat and lean broiler hens fed ad-libitum or on a controlled-feeding regime. British Poultry Science, London, v.34, p.589-596, 1993.

MAGER, W.H.; De KRUIJFF, A.J. Stress-induced transcriptional activation. Microbiological Reviews, Washington, v.59, n.3, p.506-531, 1995. Abstract.

MAHMOUD, K.Z.; EDENS, F.W. Influence of selenium sources on age related and mild heat stress-related changes of blood and liver glutathione redox cycle in broiler chickens (Gallus domesticus). Comparative Biochemistry and Physiology - Part B: Biochemistry \& Molecular Biology, Vancouver, v.136, n.4, p.921-934, 2003. Abstract.

McKEE, S.R.; SAMS, A.R. Rigor mortis development at elevated temperatures induces pale exudative turkey meat characteristics. Poultry Science, Ithaca, v.77, p.169-174, 1998.

MITCHELL, M.A.; KETTLEWELL, P.J. Physiological stress and welfare of broiler chickens in transit: solutions not problems! Poultry Science, Ithaca, v.77, p.1803-1814, 1998.

OFFER, G.; KNIGHT, P. The structural basis of water holding in meat. Part 2: drip losses. In: LAWRIE, R. Developments in meat science-4. London: Elsevier Applied Science, 1988. Chap.4, p.172-243.

OLANREWAJU, H. A. et al. Stress and acid-base balance in chickens. Poultry Science, Ithaca, v.85, p.1266-1274, 2006

OLIVEIRA, R. et al. Efeitos da temperatura e da umidade relativa sobre o desempenho e o rendimento de cortes nobres de frangos de corte de 1 a 49 dias de idade. Revista Brasileira de Zootecnia, Viçosa, v.35, n.3, p.797-803, 2006.

PATIENCE, J.F. A review of the role of acid-base balance in amino acid nutrition. Journal of Animal Science, Albany, v.68, p.398-408, 1990
PETRACCI, M. et al. The effect of holding temperature on live shrink, processing yield, and breast meat quality of broiler chickens. Poultry Science, Ithaca, v.80, p.670-675, 2001.

SAMS, A.R.; MILLS, K.A. The effect of feed withdrawal duration on the responsiveness of broiler pectoralis to rigor mortis acceleration. Poultry Science, Ithaca, v.72, n.9, p.1789-1796, 1993.

SANDERCOCK, D.A. et al. Physiological responses to acute heat stress in broilers: implications for meat quality? In: EUROPEAN SYMPOSIUM ON THE QUALITY OF POULTRY MEAT, 14., 1999, Bologna. Proceedings... Bologna: World's poultry Science Association, 1999. p.271276.

SANDERCOCK, D.A. et al. Acute heat stress-induced alterations in blood acid-base status and skeletal muscle membrane integrity in broiler chickens at two ages: Implications for meat quality. Poultry Science, Ithaca, v.80, p.418-425, 2001.

SANDERCOCK, D.A. et al. Metabolic heat production in fast and slow growing broiler chickens during acute heat stress. British Poultry Science, London, v.36, p.868, 1995.

SWATLAND, H.J. On line evaluation of meat. Lancaster: Technomic, 1995. 347p.

TANKSON, J.D. et al. Stress and nutritional quality of broilers. Poultry Science, Ithaca, v.80, n.9, p.1384-1389, 2001.

TEETER, R.G. et al. Chronic heat stress and respiratory alkalosis: occurrence and treatment in broiler chickens. Poultry Science, Ithaca, v.64, p.1060-1064, 1985.

TINÔCO, I.F.F. Ambiência e instalações para a avicultura industrial. In: ENCONTRO NACIONAL DE TÉCNICOS, PESQUISADORES E EDUCADORES DE CONSTRUÇÕES RURAIS, 3., 1998, Poços de Caldas, MG. Anais... Poços de Caldas: Sociedade Brasileira de Engenharia Agrícola, 1998. p.186

VERCELLOTTI, J.R. et al. Lipid oxidation in foods: An overview. In: St. ANGELO. Lipid oxidation in food. Washington: American Chemical Society, 1992. p.1-11.

WOELFEL, R.L. et al. The characterization and incidence of pale, soft, and exudative broiler meat in a commercial processing plant. Poultry Science, Ithaca, v.81, p.579-584, 2002.

YAHAV, S. et al. Blood system responses of chickens to changes in environmental temperature. Poultry Science, Ithaca, v.76, p.627-633, 1997. 